\title{
Influence of fluid deprivation level on the extinction of conditioned taste aversion induced by amphetamine in female rats
}

\author{
PAUL J. WELLMAN \\ Texas A\&M University, College Station, Texas 77843 \\ and \\ CHRISTOPHER G. BOISSARD \\ Bucknell University, Lewisburg, Pennsylvania 17837
}

\begin{abstract}
The present experiment examined the influence of differential fluid deprivation (8.5- or 23.5-h) on the extinction of amphetamine $(2.0 \mathrm{mg} / \mathrm{kg})$-induced conditioned taste aversion in female rats. In two-bottle preference tests, rats trained and extinguished under a 23.5-h fluid-deprivation schedule displayed significantly faster extinction of saccharin taste aversion relative to that observed in rats trained and extinguished under an 8.5-h deprivation schedule. The more rapid extinction of amphetamine aversion observed in fluid-deprived rats is comparable to that described previously for conventional emetic punishers such as lithium chloride or cyclophosphamide.
\end{abstract}

Conditioned taste aversion (CTA) refers to the decreased consumption of a novel food or flavored solution previously paired with an illness experience (Garcia, Ervin, \& Koelling, 1966). A variety of substances, including apomorphine, cyclophosphamide, lithium chloride, amphetamine, scopolamine, morphine, and $\Delta^{9}$-THC, are known to produce taste aversion learning (Berger, 1972; Cappell, LeBlanc, \& Endrenyi, 1973; Lorden, Callahan, \& Dawson, 1980; Sklar \& Amit, 1977). Unconditioned stimuli such as lithium chloride are thought to induce aversion via conditioning of their emetic properties. Taste aversion induced by lithium chloride is attenuated by pretreatment with antiemetic drugs (Coil, Hankins, Jenden, \& Garcia, 1978) or by lesions of the area postrema, a brainstem emetic center (McGlone, Ritter, \& Kelley, 1979). In contrast, the taste aversions induced by psychoactive drugs are thought to reflect a central action, but the precise nature of the central actions of such drugs as amphetamine is unclear (Cappell \& LeBlanc, 1977; Gamzu, 1977; Lorden et al., 1980).

Previous work has demonstrated that the magnitude and rate of extinction of conditioned taste aversion are functions of the level of fluid deprivation employed during training and extinction. Peck and

Request for reprints should be directed to the first author at the Department of Psychology, Texas A\&M University, College Station, Texas 77843. The authors would like to thank Roger M. Tarpy and Jack R. Nation for their critical reading of an early version of the manuscript.
Ader (1974), for example, reported that conditioned taste aversion induced by cyclophosphamide in rats trained and extinguished under deprivation or satiation schedules was significantly stronger under a water-satiation condition than under a 23.5 -h fluiddeprivation condition. With regard to rate of extinction, Grote and Brown (1973) and Sengstake, Chambers, and Thrower (1978) found that taste aversion induced by either lithium chloride or cyclophosphamide extinguished at a more rapid rate in highly fluid-deprived animals.

The relationship between level of fluid deprivation and rate of extinction of conditioned taste aversion, however, has been delineated only for conventional punishers such as lithium chloride and cyclophosphamide. Inasmuch as the pharmacological and neuroanatomical bases of taste aversions induced by emetic and psychoactive agents such as amphetamine are known to be independent (cf. McGlone, Ritter, \& Kelley, 1979; Sklar \& Amit, 1977; Wellman, McIntosh, \& Guidi, 1981), it is unclear whether accelerated extinction of psychoactive taste aversions would be obtained under conditions of fluid deprivation. The present experiment therefore extends the generality of the fluid-deprivation effect to psychoactive agents by demonstrating that female rats trained and extinguished under a 23.5-h fluid-deprivation schedule display faster extinction of an amphetamineinduced conditioned taste aversion than do rats trained and extinguished under an 8.5-h fluiddeprivation schedule. 


\section{METHOD}

Subjects

The subjects were 52 female Long-Evans hooded rats (Blue Spruce Farms, Inc.), approximately 80 days of age at the beginning of the experiment. All rats were individually housed in standard wire-mesh cages in a temperature-controlled $\left(23^{\circ}-25^{\circ} \mathrm{C}\right)$ colony room under a 12-h light cycle (lights on at $0800 \mathrm{~h}$ ). A pellet diet (Wayne) was freely available in the home cage throughout the experiment, whereas water was available as described in the schedules below.

\section{Drugs}

A saline solution consisted of $.9 \%(w / v)$ sodium chloride (Fisher) dissolved in sterile distilled water. Amphetamine solutions were prepared prior to injection by dissolving dextro-amphetamine sulfate $(2.0 \mathrm{mg} / \mathrm{ml})$ into the saline solution. All solutions were calculated as the salt.

\section{Procedure}

The rats were assigned randomly to one of four groups of a $2 \times 2$ factorial design with drug $(.9 \%$ saline or $2.0 \mathrm{mg} / \mathrm{kg}$ amphetamine) and water-deprivation schedule $(8.5$ or $23.5 \mathrm{~h})$ as the factors. Twenty rats were deprived of water during an 8.5 -h period prior to each intake test and were given free access to water in the home cage during the 15 -h interval following each intake test. The remaining 32 rats were placed on a $23.5-\mathrm{h}$ waterdeprivation schedule. Drinking tests were conducted in separate drinking cages and were $30 \mathrm{~min}$ in length. Pellets were freely available in the home cages but not in the drinking cages.

Baseline water intakes were recorded for each rat on 9 consecutive days. On Day 10, the rats were offered a .1\% saccharin solution in place of water during a 30 -min period. Immediately following saccharin consumption, each rat was injected (ip) with either saline or amphetamine $(1.0 \mathrm{ml} / \mathrm{kg})$ and returned to the home cage. During Days 11-15, the extinction phase of the experiment, the deprivation schedules were carried out as before and each rat was given daily access to both $.1 \%$ saccharin and water. Bottle position was alternated daily to prevent position preference, and fluid intakes were measured to the nearest $1.0 \mathrm{ml}$ from calibrated Wahmann tubes.

\section{Statistical Analyse}

Split-plot analyses of variance (Kirk, 1968) were computed for the intake data, after conversion to standard suppression ratios (saccharin/total consumption). All statistical tests were made at the .05 level of significance.

\section{RESULTS}

Because examination of the saccharin intakes on the training day revealed that several animals in the 8.5-h fluid-deprivation condition consumed no saccharin, data were analyzed for only those rats that consumed at least $1.0 \mathrm{ml}$ of the saccharin solution. Data from seven rats in the 8.5-h deprivation condition were discarded from the experiment because these animals failed to meet this criterion.

Figure 1 depicts the changes in saccharin intake, expressed as standard suppression ratios, produced by the deprivation and drug treatments of this experiment. Analyses of variance revealed significant main effects of drug $[F(1,41)=83.0]$, deprivation $[F(1,41)=3.1]$, and day $[F(4,164)=5.9]$. Only the deprivation $\times$ drug interaction was significant $[F(1,41)$ $=4.0]$. These analyses suggest that the saccharin in-

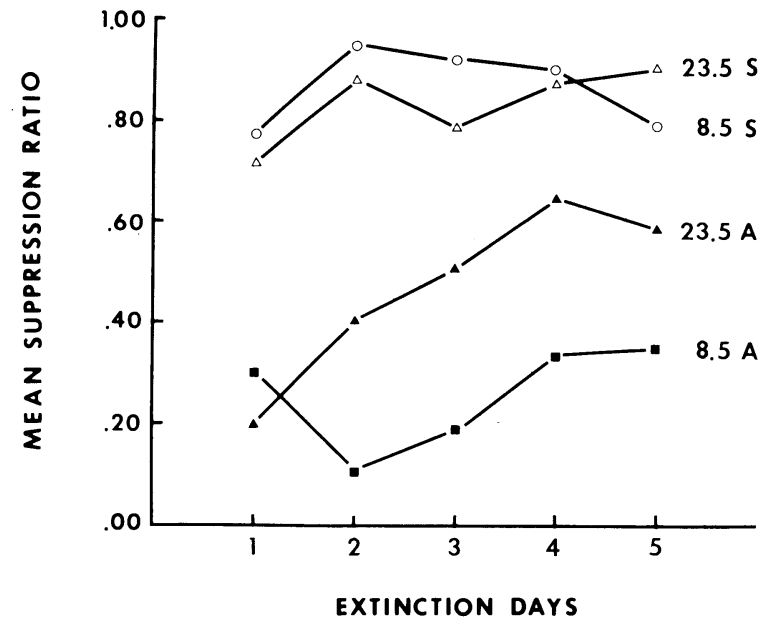

Figure 1. Group mean daily suppression ratios (saccharin intake/ saccharin plus water intake) for female rats treated on the training day with either $.9 \%$ saline (S) or $\mathbf{2 . 0} \mathrm{mg} / \mathrm{kg}$ dextro-amphetamine sulfate (S). For all rats, training and extinction testing were carried out under either an 8.5-h or a 23.5-h fluid-deprivation schedule.

takes of rats treated with amphetamine on the training day were significantly suppressed during the extinction trials, relative to the saccharin intakes of rats treated with saline, and further indicate that the extinction displayed by amphetamine-treated rats was differentially influenced by the fluid-deprivation schedules used during training and extinction, as evidenced by the significant interaction between deprivation and drugs. Further analyses, using group suppression ratios collapsed across the five daily extinction periods, demonstrated that rats trained and extinguished under a 23.5-h deprivation schedule and treated with amphetamine displayed significantly faster extinction than did rats trained under an 8.5-h deprivation schedule $[\mathrm{t}(41)=2.1]$.

Water deprivation produced differential saccharin intake on the training day (see Table 1), with the 23.5-h water-deprived rats consuming significantly more saccharin than the 8.5 -h water-deprived rats $[t(43)=3.8]$. It is unlikely that these differential intakes contributed to the differential extinction observed in amphetamine-treated rats, because the relationship between amount of CS consumed and magnitude of conditioning is typically observed to be. a direct relationship (e.g., greater amounts of CS consumed result in stronger aversion conditioning; Bond \& Harland, 1975). To discount this notion, however, further analyses of variance were made of the percent changes in saccharin intake from Day 10 baseline saccharin intake for each of the five extinction periods (see Table 1). Saline-treated rats displayed progressively larger saccharin intakes over the 5 extinction days, with the 8.5 -h-deprived rats displaying larger increases in saccharin consumption than the 23.5-h-deprived rats $[F(1,41)=7.0]$. This finding, however, probably reflects asymptotic levels 
Table 1

Group Mean Saccharin Intake (SI) on Day 10 and SI, Water Intake (WI), and Percent Change (PC) from Day 10 Saccharin Baseline Values on Day s 1 1-15 for Amphetamine- $(2.0 \mathrm{mg} / \mathrm{kg})$ and Saline-Treated Rats Trained and Extinguished on Either an 8.5-h or a 23.5-h Water-Deprivation Schedule

\begin{tabular}{|c|c|c|c|c|c|c|c|c|c|c|c|c|c|c|c|c|c|c|}
\hline & \multicolumn{3}{|c|}{ Day $10 \mathrm{SI}$} & \multicolumn{3}{|c|}{ Day 11} & \multicolumn{3}{|c|}{ Day 12} & \multicolumn{3}{|c|}{ Day 13} & \multicolumn{3}{|c|}{ Day 14} & \multicolumn{3}{|c|}{ Day 15} \\
\hline & $\mathrm{N}$ & Mean & SEM & SI & WI & $\mathrm{PC}$ & SI & WI & PC & SI & WI & PC & SI & WI & $\mathrm{PC}$ & SI & WI & PC \\
\hline & \multicolumn{18}{|c|}{ 8.5-h Deprivation Condition } \\
\hline Saline & 8 & 4.2 & 1.60 & 5.9 & .5 & +170.00 & 8.40 & .4 & +310.00 & 9.6 & .63 & +350 & 9.5 & .75 & +320 & 6.5 & 1.0 & +220 \\
\hline \multirow[t]{2}{*}{ Amphetamine } & 12 & 3.4 & .64 & 1.2 & 2.6 & $-\quad .44$ & .55 & 2.4 & $-\quad .42$ & 1.5 & 3.30 & -17 & 1.9 & 2.10 & -15 & 1.6 & 2.2 & -33 \\
\hline & \multicolumn{18}{|c|}{ 23-5-h Deprivation Condition } \\
\hline Saline & 10 & 9.9 & .91 & 12.6 & 4.5 & +31.00 & 14.10 & 1.9 & +52.00 & 13.7 & 1.90 & +47 & 13.7 & 1.40 & +44 & 13.5 & 1.2 & +41 \\
\hline Amphetamine & 16 & 8.9 & .79 & 2.5 & 9.9 & -74.00 & 5.50 & 7.4 & -18.00 & 7.2 & 6.90 & -17 & 10.8 & 5.00 & +18 & 8.7 & 5.8 & \\
\hline
\end{tabular}

of saccharin consumption by 23.5-h-deprived rats rather than any treatment effect. Amphetamine treatment produced significant suppressions of saccharin consumption $[F(1,41)=7.5]$, but the extinction of amphetamine aversion was again faster in the $23.5-\mathrm{h}$ deprived rats relative to that displayed by the 8.5 -hdeprived rats $[F(1,41)=4.2]$. It should be noted that this difference in suppression of saccharin consumption is not likely to be the result of an asymptotic level of consumption by the 8.5-h-deprived rats because the $8.5 \mathrm{~h}$ rats treated with saline displayed progressively larger increases in saccharin consumption over the extinction period. The percent change analyses, therefore, confirm the analyses of suppression ratios and demonstrate that the extinction of the aversion conditioning observed in amphetaminetreated rats was a function of the deprivation schedule used during training and extinction.

\section{DISCUSSION}

The outcomes of the present experiment demonstrate a direct relationship between level of fluid deprivation and the rate of extinction of amphetamine taste aversion. Specifically, rats trained and extinguished under a 23.5-h fluid-deprivation schedule displayed significantly faster extinction of amphetamine aversion than did 8.5-h fluid-deprived rats. These data therefore extend to psychoactive punishers outcomes similar to those previously reported for emetic punishers such as cyclophosphamide and lithium chloride (Grote \& Brown, 1973; Sengstake et al., 1978).

Although the neural bases of these aversions can be dissociated (e.g., McGlone et al., 1979; Wellman et al., 1978), it is clear that examination of functional relationships known to influence aversion conditioning are not sufficient to dissociate these aversions. McIntosh, Wellman, and Cain (Note 1), for example, examined the influence of CS preexposure on the aversions induced by equipotent dosages of amphetamine and of lithium chloride only to find that CS preexposure induced comparable retardation of aversion conditioning for these punishers.
Deprivation level is thought to influence the extinction of conditioned taste aversion by increasing saccharin consumption over extinction trials; more rapid extinction presumably occurs because the deprivation-induced increase in saccharin consumption either is associated with recovery from illness or serves as feedback to the animals that malaise is not forthcoming following saccharin consumption. Rate of extinction, as influenced by deprivation level, is more rapid in single-bottle than in two-bottle extinction tests. Revusky and Garcia (1970) and, more recently, Grote and Brown (1973) argue that the singlebottle extinction test actually plays thirst produced by deprivation against the acquired aversive property of the saccharin solutions: as fluid deprivation increases, so does thirst, such that at high levels of deprivation the animals must consume the CS to avoid dehydration, with enhanced saccharin consumption resulting in more rapid extinction. Although fluid deprivation also enhances saccharin consumption and rate of extinction in two-bottle tests, the magnitude of this effect is smaller than that observed when comparable levels of satiation and deprivation are used in a single-bottle test. The lesser effectiveness of fluid deprivation in enhancing saccharin consumption and rate of extinction in two-bottle tests may reflect the propensity of the animals to satisfy thirst by consuming water rather than saccharin; such a compromise is not possible in the single-bottle case.

Although thirst-induced enhancement of saccharin intake is a reasonable explanation of the deprivation effect in the single-bottle test, this explanation fails to explain why, if the animal can resolve thirst by consuming water while avoiding the saccharin CS, any enhancement of saccharin consumption and extinction of the aversion should be observed in a twobottle test. Consideration of an ancillary effect of water deprivation, that of increasing food motivation, however, may resolve this problem. Given the correlation between food and water intake (Falk, 1971), fluid deprivation not only produces a deficit in water balance, but may also induce hunger in that animals fail to eat the dry pellet diet available in 
the home cage during the 23-h deprivation interval in the absence of water. Such an analysis suggests that, during extinction tests, the animals are both thirsty and hungry. Of the several explanations relevant to this analysis, the simplest explanation would make use of data from Jacobs and Sharma (1969), who noted that food deprivation produced an enhancement of affective responsivity to palatable saccharin solutions, to suggest that increased hunger motivation alters the affective responses of the animal to the saccharin solution, resulting in increased consumption and correspondingly more rapid extinction. A more complex view might emphasize the notion that deprived animals view a saccharin solution as a food rather than as a fluid (Mook, 1974). For this view, fluid deprivation results in both thirst and hunger motivation, such that during two-bottle extinction tests, fluid-deprived rats consume water to satisfy thirst and consume saccharin to potentially satisfy hunger. In contrast, rats trained and tested under conditions of satiation are neither thirsty nor hungry and would therefore display less rapid extinction of aversion. Although admittedly tentative, the notion that fluid deprivation produces both thirst and hunger may explain the observation that fluid deprivation produces more rapid extinction of aversion in a twobottle paradigm.

\section{REFERENCE NOTE}

1. McIntosh, P., Wellman, P. J., \& Cain, N. W. The effects of CS preexposure on the acquisition and extinction of amphetamine and lithium taste aversions. Paper presented at the annual meeting of the Eastern Psychological Association, Hartford, Connecticut, 1980.

\section{REFERENCES}

BERger, B. D. Conditioning of food aversions by injections of psychoactive drugs. Journal of Comparative and Physiological Psychology, 1972, 81, 21-26.

Bond, N. W., \& Harland, W. Effect of amount of solution drunk on taste aversion learning. Bulletin of the Psychonomic Society, 1975, 5, 219-220.

Cappell, H., \& LeBlanc, A. E. Gustatory avoidance conditioning by drugs of abuse. In N. W. Milgram, L. Krames, \& T. M. Alloway (Eds.), Food aversion learning. New York: Plenum Press, 1977.
Cappell, H., LeBlanc, A. E., \& Endrenyi, L. Aversion conditioning by psychoactive drugs: Effects of morphine, alcohol and chlordiazepoxide. Psychopharmacologia, 1973, 29, 239-246.

Coil, J. D., Hankins, J. D., Jenden, D. D., \& Garcia, J. The attenuation of a specific cue-to-consequence association by antiemetic agents. Psychopharmacology, 1978, 56, 21-25.

FALK, J. L. Determining changes in vital functions: Ingestion. In R. D. Myers (Ed.), Methods in psychobiology (Vol. 1). New York: Academic Press, 1971.

Gamzu, E. The multifaceted nature of taste aversion agents: Is there a single common factor? In L. M. Barker, M. R. Best, \& M. Domjan (Eds.), Learning mechanisms in food selection. Waco, Tex: Baylor University Press, 1977.

Garcia, J., Ervin, F. R., \& Koelling, R. A. Learning with prolonged delay of reinforcement. Psychonomic Science, 1966, 5, 121-122.

Grote, F. W., \& Brown, R. T. Deprivation level affects extinction of a conditioned taste aversion. Learning and Motivation, 1973, 4, 314-319.

Jacobs, H. L., \& Sharma, K. N. Taste versus calories: Sensory and metabolic signals in the control of food intake. Annals of the New York Academy of Sciences, 1967, 157 (R2), 1084-1125.

KIRK, R. E. Experimental design: Procedures for the behavioral sciences. Belmont, Calif: Brooks/Cole, 1968.

Lorden, J. F., Callahan, M., \& Dawson, R. Depletion of central catecholamines alters amphetamine- and fenfluramineinduced taste aversions in the rat. Journal of Comparative and Physiological Psychology, 1980, 94, 99-114.

McGlone, J. J., RitTe R, S., \& Kelly, K. Area postrema lesion eliminates the antiaggressive and aversive conditioning properties of lithium. Neuroscience Abstracts, 1979, 5, 655.

Моoк, D. G. Saccharin preference in the rat: Some unpalatable findings. Psychological Review, 1974, 81, 475-490.

Peck, J. H., \& Ader, R. Illness-induced taste aversion under states of deprivation and satiation. Animal Learning \& Behavior, 1974, 2, 6-8.

REvUSKY, S., \& GARCIA, J. Learned associations over long delays. In G. H. Bower (Ed.), The psychology of learning and motivation: Advances in theory and research (Vol. 4). New York: Academic Press, 1970.

Sengstake, C. B., Chambers, K. C., \& Thrower, J. H. Interactive effects of fluid deprivation and testosterone on the expression of a sexually dimorphic conditioned taste aversion. Journal of Comparative and Physiological Psychology, 1978, 2, 1150-1155.

SklaR, L. J., \& Aмıт, Z. Manipulation of catecholamine systems blocks the conditioned taste aversion induced by self-administered drugs. Neuropharmacology, 1977, 16, 649-655.

Wellman, P. J., McIntosh, P., \& Guidi, E. Effects of dorsolateral tegmental lesions on amphetamine- and lithium-induced taste aversions. Physiology \& Behavior, 1981, 26, 341-344.

(Manuscript received April 14, 1981; revision accepted for publication July 16,1981 .) 